Virginia Commonwealth University VCU Scholars Compass

2014

\title{
First-principles studies on graphene-supported transition metal clusters
}

\author{
Sanjubala Sahoo \\ Virginia Commonwealth University, ssahoo2@vcu.edu \\ Markus E. Gruner \\ University of Duisburg-Essen \\ Shiv N. Khanna \\ VirginiaCommonwealth University, snkhanna@vcu.edu \\ Peter Entel \\ University of Duisburg-Essen
}

Follow this and additional works at: http://scholarscompass.vcu.edu/phys_pubs

Part of the Physics Commons

Sahoo, S., Gruner, M. E., \& Khanna, S. N., et al. First-principles studies on graphene-supported transition metal clusters. The Journal of Chemical Physics, 141, 074707 (2014). Copyright (C) 2014 AIP Publishing LLC.

\section{Downloaded from}

http://scholarscompass.vcu.edu/phys_pubs/103

This Article is brought to you for free and open access by the Dept. of Physics at VCU Scholars Compass. It has been accepted for inclusion in Physics Publications by an authorized administrator of VCU Scholars Compass. For more information, please contact libcompass@vcu.edu. 


\title{
First-principles studies on graphene-supported transition metal clusters
}

\author{
Sanjubala Sahoo, ${ }^{1, a)}$ Markus E. Gruner, ${ }^{2}$ Shiv N. Khanna, ${ }^{1}$ and Peter Entel ${ }^{2}$ \\ ${ }^{1}$ Department of Physics, Virginia Commonwealth University, Richmond, Virginia 23284, USA \\ ${ }^{2}$ Faculty of Physics and Center for Nanointegration (CENIDE), University of Duisburg-Essen, \\ 47048 Duisburg, Germany
}

(Received 7 May 2014; accepted 4 August 2014; published online 20 August 2014)

\begin{abstract}
Theoretical studies on the structure, stability, and magnetic properties of icosahedral $\mathrm{TM}_{13}(\mathrm{TM}=\mathrm{Fe}$, $\mathrm{Co}, \mathrm{Ni}$ ) clusters, deposited on pristine (defect free) and defective graphene sheet as well as graphene flakes, have been carried out within a gradient corrected density functional framework. The defects considered in our study include a carbon vacancy for the graphene sheet and a five-membered and a seven-membered ring structures for graphene flakes (finite graphene chunks). It is observed that the presence of defect in the substrate has a profound influence on the electronic structure and magnetic properties of graphene-transition metal complexes, thereby increasing the binding strength of the $\mathrm{TM}$ cluster on to the graphene substrate. Among $\mathrm{TM}_{13}$ clusters, $\mathrm{Co}_{13}$ is absorbed relatively more strongly on pristine and defective graphene as compared to $\mathrm{Fe}_{13}$ and $\mathrm{Ni}_{13}$ clusters. The adsorbed clusters show reduced magnetic moment compared to the free clusters. ( 2014 AIP Publishing LLC. [http://dx.doi.org/10.1063/1.4893328]
\end{abstract}

\section{INTRODUCTION}

Graphene is a promising substrate material due to the inertness. ${ }^{1-3}$ Its unique electronic structure,,${ }^{4,5}$ such as a pointlike Fermi surface and linear dispersion of bands near the Fermi level, renders it as a unique material for studying ballistic transport and quantum Hall effect. ${ }^{6}$ It can act as a versatile substrate material for nano electronics. ${ }^{7}$ It is an attractive material for efficient extraction of light over a wide range of wavelengths enabling for optoelectronic applications. ${ }^{8}$ The photoconductivity of graphene is altered by the adsorption of atmospheric gases. ${ }^{9}$ This property can be used to develop gas sensors. Apart from these fascinating properties, deposited transition metal clusters on graphene show high catalytic activity. ${ }^{10-14}$ Several experimental studies also exist for the chemical activities of $\mathrm{Ni},{ }^{15} \mathrm{Co},{ }^{16} \mathrm{Pt},{ }^{17-21} \mathrm{Pd},{ }^{22}$ and $\mathrm{Rh}^{23}$ clusters on different carbon supports.

Although several studies exist for the magnetic properties of graphene-supported clusters of $4 d$ or $5 d$ elements such as $\mathrm{Pd}$ or Pt, such studies are still quite limited for clusters of ferromagnetic $3 d$ elements such as $\mathrm{Fe}, \mathrm{Co}$, and $\mathrm{Ni}$. In particular, there are few studies on magneto-crystalline anisotropy that is critical for magnetic storage devices. ${ }^{24,25}$ It has been shown that Co dimers adsorbed on graphene show a large magnetic anisotropy. ${ }^{24}$ In spite of the fact that many theoretical studies based on density functional theory (DFT) exist for graphene supported $\mathrm{Fe}$, Co, and $\mathrm{Ni}$ adatoms and dimers ${ }^{26-29}$ studies for still larger clusters are limited. ${ }^{30,31}$ For example, Johll et ll $^{30}$ and Longo et al. ${ }^{32}$ have studied the structural, magnetic, and electronic properties of the graphene supported Fe, $\mathrm{Co}$, and Ni clusters only up to 4 atoms. In this work, we deal with still larger clusters consisting of 13 atoms supported on graphene.

a)E-mail: ssahoo2@vcu.edu
It has been reported that presence of carbon vacancies in graphene modifies the physical and chemical properties of graphene significantly. ${ }^{33-36}$ In particular, it has been observed that defect sites act as pinning centers and possess high adsorption energies. ${ }^{37}$ This property can be used to manipulate materials at atomistic scale; for example, Wang et al. have used defect sites of graphene for controlled growth of $\mathrm{Fe}$, $\mathrm{Co}$, and $\mathrm{Ni}$ nano particles ${ }^{38}$ in their experiments. In fact, due to the two-dimensional hexagonal structure, graphene shows several kinds of topological defects ${ }^{39}$ such as pentagonal defects, i.e., a local five-membered ring structure (5-m), heptagonal defects, i.e., a local seven-membered ring structure (7-m), or a combination of both, known as the Stone-Wales defect. ${ }^{40,41}$ Using tight-binding molecular dynamics simulations Lee et $a l .{ }^{42}$ have studied the diffusion, coalescence, and reconstruction of these defects and find that the coalescence of two single vacancies into a double vacancy and thus StoneWales type reconstructions are probabilistic. Their calculations also show that four single vacancies reconstruct into two collective 555-777 defects. In view of all these reports, it is worthwhile to study the influence of vacancy type defects in graphene on the properties of deposited clusters.

In the present study, we have placed the closed geometric shell $\mathrm{TM}_{13}(\mathrm{TM}=\mathrm{Fe}, \mathrm{Co}$, and $\mathrm{Ni})$ clusters on both pristine and defective graphene substrates and performed a detailed investigation of the resulting change in geometry, magnetism, and electronic properties of the clusters as well as the substrate. The paper is organized as follows. In Sec. II, we discuss the theoretical method used for our calculations. The results and discussions are in Sec. III which is divided into three subsections. The qualitative features of free $\mathrm{TM}_{13}$ clusters are discussed in Sec. III A. Section III B contains results of $\mathrm{TM}_{13}$ clusters adsorbed on pristine and defective graphene sheet with a carbon vacancy. Section III C contains results on $\mathrm{TM}_{13}$ clusters adsorbed on pristine, 5- $m$ and 7- $m$ type 
defective graphene flakes. Finally, in Sec. IV, the results are summarized.

\section{COMPUTATIONAL METHODS}

Calculations have been performed within the framework of DFT using the generalized gradient approximation (GGA). The Perdew-Burke-Ernzerhof (PBE) exchangecorrelation functional $^{43}$ is used for the exchange and correlation potential. The calculations are performed using the Vienna $a b$ initio simulation package (VASP), ${ }^{4,45}$ which uses a plane wave basis. The interactions between the core and valence electrons are treated with the projector augmented wave method. ${ }^{46}$ The $\mathrm{TM}_{13}$ clusters are adsorbed on pristine graphene sheet which consists of a supercell constructed from $5 \times 3$ times the primitive cell of hexagonal graphene and contains $60 \mathrm{C}$ atoms. Periodic boundary condition is applied along two directions of the planar graphene supercell while the direction perpendicular to the graphene is treated with open boundary condition by selecting a large vacuum in order to minimize the image interactions. The geometry optimization is done using the conjugate gradient method. The integration in the Brillouin zone is done through a $2 \times 2 \times 1$ $k$-points mesh. The self-consistent field calculations are carried out with the energy convergence criterion of $10^{-6} \mathrm{eV}$. The electronic density of states (DOS) are calculated with Gaussian broadening parameter of $0.05 \mathrm{eV}$ and a $k$-points mesh of $11 \times 11 \times 1$ for graphene sheet.

Graphene flakes (finite chunks of graphene) can be treated as a zero-dimensional system and hence supercell calculations similar to clusters can be performed. In case of the pristine, 5- $m$ and 7- $m$ graphene flakes we used hydrogenterminated clusters $\mathrm{C}_{54} \mathrm{H}_{18}, \mathrm{C}_{45} \mathrm{H}_{15}$, and $\mathrm{C}_{63} \mathrm{H}_{21}$, respectively. A supercell size of $23 \times 23 \times 15 \AA^{3}$ is used for pristine and 5 - $m$ graphene flakes and $26 \times 26 \times 15 \AA^{3}$ supercell size is used for $7-m$ graphene flake in order to minimize the interaction between the periodic images. The cluster calculations are done at the $\Gamma$-point of the Brillouin zone.

\section{RESULTS AND DISCUSSION}

\section{A. Properties of $\mathrm{TM}_{13}$ clusters}

The closed shell clusters with icosahedral geometry are highly symmetric and show enhanced stability compared to other geometries. ${ }^{47-50}$ Thus, we have selected these closedshell $\mathrm{TM}_{13}$ clusters for our studies to adsorb on graphene. The structures chosen for the studies have the center-to-shell distances $\left(d_{\text {Center-shell }}\right) 2.39 \AA, 2.33 \AA$, and $2.32 \AA$ for $\mathrm{Fe}_{13}, \mathrm{Co}_{13}$, and $\mathrm{Ni}_{13}$, respectively, which gives the minimum energy. It has been reported earlier that $\mathrm{Fe}_{13}$ has two magnetic states with energy separation of $29 \mathrm{meV} .^{25,51}$ The high-spin state has a magnetic moment of $44 \mu_{\mathrm{B}}$ /cluster (ferromagnetic), while the low-spin state has a magnetic moment of $34 \mu_{\mathrm{B}}$ /cluster, where the center $\mathrm{Fe}$ atom has magnetic moment antiparallel to that of the twelve neighboring $\mathrm{Fe}$ atoms. ${ }^{52}$ In contrast to $\mathrm{Fe}_{13}$, only one spin state with ferromagnetic alignment for all sites is found for $\mathrm{Co}_{13}\left(31 \mu_{\mathrm{B}}\right)$ and $\mathrm{Ni}_{13}\left(8 \mu_{\mathrm{B}}\right)$. The high spin state of $\mathrm{Fe}_{13}$ with $44 \mu_{\mathrm{B}}$ was found to be more stable and has been used for the studies of adsorption of $\mathrm{Fe}_{13}$ cluster on graphene sheets and graphene flakes as discussed in the following subsections.

\section{B. $\mathrm{TM}_{13}$ clusters on graphene sheet}

The cluster with icosahedral geometry can be adsorbed on the graphene sheet with three possible orientations, namely, the vertex (top of atom) facing graphene, the bonding edge of surface atoms (bond site) facing graphene, or the triangular facet facing the substrate. Among these configurations, for $\mathrm{Fe}_{13}$ and $\mathrm{Ni}_{13}$ on graphene, the lowest energy structure has the triangular facet of the cluster facing the graphene, whereas for $\mathrm{Co}_{13}$ on graphene an orientation with the edge site of the cluster facing the graphene sheet is found to be energetically most favorable. The optimized geometries for the stable configurations are shown in Fig. 1. A complete relaxation for all atoms of $\mathrm{TM}_{13}$ clusters deposited on graphene results in distortion of the icosahedral geometry with a slight appearance of the local distortion on the graphene substrate (approximately $1.4 \%$, i.e., the $\mathrm{C}-\mathrm{C}$ bond length increases from $1.43 \AA$ to $1.45 \AA$ ).

Among three types of $\mathrm{TM}_{13}$ clusters considered, the maximum distortion is observed for adsorbed $\mathrm{Co}_{13}$, where the center-shell distance (cluster radius) varies from $2.30 \AA$ to $2.51 \AA\left(\Delta d_{\text {Center-shell }}=0.21 \AA\right)$ as compared to that of the adsorbed $\mathrm{Fe}_{13}$ and $\mathrm{Ni}_{13}$ on graphene with $\Delta d_{\text {Center-shell }}$ values of $0.14 \AA$ and $0.12 \AA$, respectively. The average center-shell distances for $\mathrm{TM}_{13}$ clusters are reported in Table $\mathrm{I}$.

Analysis of the TM-C bonds shows that three facet $\mathrm{Fe}$ atoms of the $\mathrm{Fe}_{13}$ cluster facing the graphene sheet are bonded to six carbon atoms of a hexagonal ring of the graphene sheet. On the other hand for $\mathrm{Co}_{13}$, four Co atoms facing the graphene sheet have a tendency to form bonds with the $\mathrm{C}$ atoms. The orientation of the relaxed $\mathrm{Co}_{13}$ is such that one of the four Co atoms is bonded to six $\mathrm{C}$ atoms of a hexagonal ring of graphene, with the average $\mathrm{Co}-\mathrm{C}$ bond length of $2.15 \AA$. As a result of bonding of $\mathrm{Co}$ to several $\mathrm{C}$ atoms, the magnetic moment of the Co atom is reduced to $0.82 \mu_{\mathrm{B}}$, while the average magnetic moment for rest twelve Co atoms amounts to $1.68 \mu_{\mathrm{B}}$. The average $\mathrm{Co}-\mathrm{C}$ bond length of $\mathrm{Co}$ atom bonded

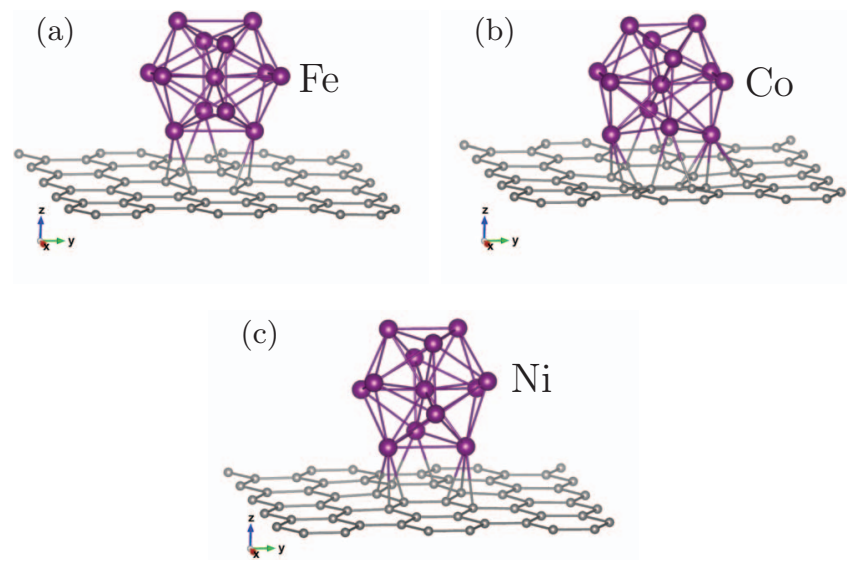

FIG. 1. Optimized structures of $\mathrm{Fe}_{13}$ (a), $\mathrm{Co}_{13}$ (b), and $\mathrm{Ni}_{13}$ (c) clusters on pristine graphene sheet. The dark (purple) and the light (grey) balls represent the TM and $\mathrm{C}$ atoms, respectively. 
TABLE I. Table showing the adsorption energy $E_{\text {Ads }}(\mathrm{eV})$, magnetic moments $\left(\mu_{\mathrm{B}}\right)$, center to shell distance of the cluster $\left(d_{\text {Center-shell }}\right)$ in $\AA$, and the Bader charges $\left(q^{\mathrm{B}}\right)$ of elemental species. $\mathrm{M}_{\mathrm{Tot}},\left\langle\mathrm{M}_{\mathrm{TM}}\right\rangle$, and $\left\langle\mathrm{M}_{\mathrm{C}}\right\rangle$ denote the total magnetic moments, average magnetic moment on $\mathrm{TM}_{13}$, and average magnetic moment on $\mathrm{C}$ atoms, respectively. $\left\langle d_{\text {Center-shell }}\right\rangle$ is the average center to shell distance for $\mathrm{TM}_{13}$ clusters. $\left\langle q_{\mathrm{C}}^{\mathrm{B}}\right\rangle$ represents the average $\mathrm{Bader}$ charge of $\mathrm{TM}$ atom in the cluster and on the $\mathrm{C}$ atom in the graphene, respectively. The number of electrons treated as valence electrons are 8, 9, 10, and 4 for $\mathrm{Fe}, \mathrm{Co}, \mathrm{Ni}$, and $\mathrm{C}$, respectively.

\begin{tabular}{|c|c|c|c|c|c|c|c|c|c|}
\hline \multirow[b]{2}{*}{ TM } & \multirow[b]{2}{*}{ Case } & \multirow[b]{2}{*}{ No. of electrons } & \multirow[b]{2}{*}{$E_{\text {Ads }}$} & \multicolumn{3}{|c|}{ Magnetic moment } & \multirow[b]{2}{*}{$\left\langle d_{\text {Center-shell }}\right\rangle$} & \multicolumn{2}{|c|}{ Bader charges $(e)$} \\
\hline & & & & $\mathrm{M}_{\mathrm{Tot}}$ & $\left\langle\mathrm{M}_{\mathrm{TM}}\right\rangle$ & $\left\langle\mathrm{M}_{\mathrm{C}}\right\rangle$ & & $\left\langle q_{\mathrm{TM}}^{\mathrm{B}}\right\rangle$ & $\left\langle q_{\mathrm{C}}^{\mathrm{B}}\right\rangle$ \\
\hline \multirow[t]{5}{*}{$\mathrm{Fe}$} & Pristine sheet & 344 & 0.882 & 39 & 2.789 & 0.019 & 2.399 & 7.919 & 4.174 \\
\hline & Defect sheet & 340 & 6.837 & 36 & 2.578 & 0.030 & 2.391 & 7.884 & 4.163 \\
\hline & Pristine flake & 338 & 0.667 & 40 & 2.837 & 0.021 & 2.397 & 7.943 & 4.087 \\
\hline & 5- $m$ flake (concave) & 299 & 2.807 & 35 & 2.594 & 0.010 & 2.412 & 7.849 & 4.112 \\
\hline & 7- $m$ flake & 377 & 2.502 & 37 & 2.681 & 0.021 & 2.390 & 7.898 & 4.107 \\
\hline \multirow[t]{5}{*}{ Co } & Pristine sheet & 357 & 2.144 & 21 & 1.618 & 0.010 & 2.367 & 8.914 & 4.116 \\
\hline & Defect sheet & 353 & 7.998 & 19 & 1.490 & 0.019 & 2.336 & 8.886 & 4.138 \\
\hline & Pristine flake & 351 & 2.021 & 21 & 1.614 & 0.010 & 2.366 & 8.916 & 4.081 \\
\hline & 5- $m$ flake (concave) & 312 & 4.307 & 22 & 1.661 & 0.010 & 2.374 & 8.884 & 4.109 \\
\hline & 7- $m$ flake & 390 & 3.716 & 24 & 1.709 & 0.009 & 2.334 & 8.914 & 4.089 \\
\hline \multirow[t]{5}{*}{$\mathrm{Ni}$} & Pristine sheet & 370 & 1.772 & 8 & 0.647 & 0.003 & 2.348 & 9.951 & 4.145 \\
\hline & Defect sheet & 366 & 7.421 & 6 & 0.489 & 0.006 & 2.357 & 9.911 & 4.112 \\
\hline & Pristine flake & 364 & 1.603 & 8 & 0.648 & 0.004 & 2.349 & 9.957 & 4.164 \\
\hline & 5-m flake (convex) & 325 & 2.973 & 9 & 0.696 & 0.001 & 2.341 & 9.955 & 4.119 \\
\hline & 7- $m$ flake & 403 & 3.737 & 7 & 0.573 & 0.006 & 2.354 & 9.941 & 4.081 \\
\hline
\end{tabular}

to three $\mathrm{C}$ atoms is the smallest with the value of $2.09 \AA$, while the two Co atoms bonded to two $\mathrm{C}$ atoms of graphene have an average bond length of $2.22 \AA$. On the other hand, the adsorbed $\mathrm{Ni}$ cluster on graphene sheet exhibits a similar structural pattern as observed for Fe. Details of the bonding feature can be seen in Fig. 1. The $\mathrm{C}$ atoms which are close to the cluster slightly move from their positions in the direction perpendicular to the surface thereby generating a slightly concave (carbon atoms move away from the cluster) or convex (carbon atoms move towards the cluster) topology. The maximum displacement of the carbon atoms is observed for Co adsorbtion, where the carbon atoms move away from the cluster by almost $0.44 \AA$, similarly for Fe adsorption the carbon atoms move by $0.18 \AA$, both tending to form concave geometry. On the other hand, $\mathrm{Ni}$ adsorption leads to a convex topology, where the carbon atoms move almost $0.23 \AA$ towards the Ni cluster. This observation is also reflected for 5- $m$ graphene flake, as is discussed in Sec. III C in the following.

The binding strength between the cluster and graphene is given by the adsorption energy $\left(E_{\mathrm{Ads}}\right)$, which is defined as

$$
E_{\mathrm{Ads}}=E_{\mathrm{Tot}}^{\text {Cluster }}+E_{\mathrm{Tot}}^{\text {Graphene }}-E_{\mathrm{Tot}}^{(\text {Cluster+Graphene) }} .
$$

Here, $E_{\mathrm{Tot}}^{\text {Cluster }}$ is the total energy of free TM cluster, $E_{\mathrm{Tot}}^{\text {Graphene }}$ is the total energy of (pristine or defective) graphene sheet or graphene flake, and $E_{\mathrm{Tot}}^{\text {(Cluster+Graphene) }}$ is the total energy of the cluster adsorbed on (pristine or defective) graphene sheet or graphene flake. The $E_{\mathrm{Ads}}$ and magnetic moments for the deposited clusters are presented in Table I. Our results show large $E_{\text {Ads }}$ for $\mathrm{Co}_{13}$ followed by $\mathrm{Ni}_{13}$ clusters as compared to that of $\mathrm{Fe}_{13}$. This indicates that $\mathrm{Fe}_{13}$ is weakly bound to the pristine graphene. Similar tendency has been also observed for $\mathrm{Fe}$ adatom on pristine graphene. ${ }^{28}$
In order to study the adsorption of clusters on defective graphene, we first introduced point defect to the graphene sheet only with a single carbon vacancy in the lattice. The substrate when relaxed show local reconstruction of atoms around the defect for the atoms $\mathrm{A}, \mathrm{B}$, and $\mathrm{C}$ as shown in Fig. 2(a). The structural distortion has characteristics of the Jahn-Teller (JT) effect, ${ }^{53}$ where the system undergoes structural distortions accompanied by the removal of degeneracy of electronic states at the Fermi level. It must be emphasized that we have found two different types of JT distortions with different relaxation settings. In one case a constrained
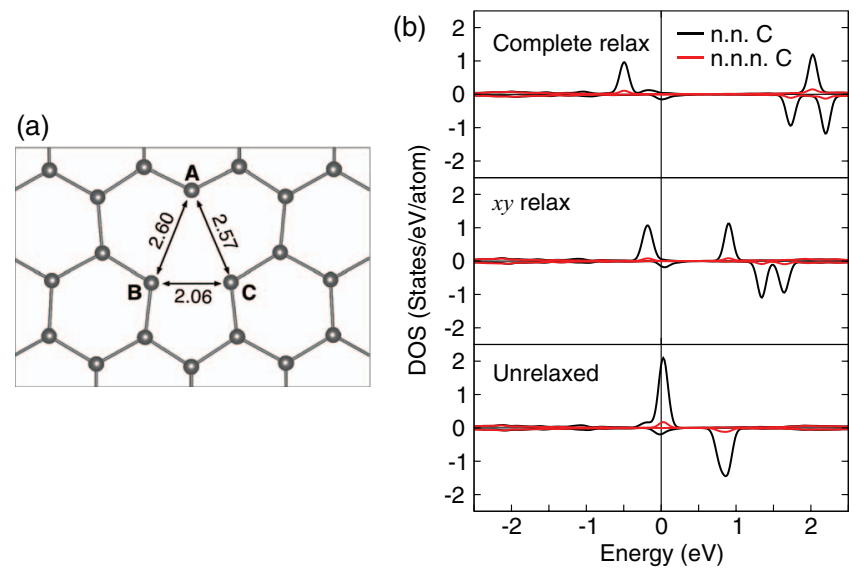

FIG. 2. (a) The local relaxation of graphene sheet with a monovacancy obtained from our calculations, where the grey balls represent the carbon atoms. The relative bond lengths of carbon atoms in close proximity to the vacancy and marked as A, B, and C are shown in Table II. (b) The density of states (DOS) for the unrelaxed (lower panel), relaxation constrained to $x y$-plane (middle panel), and free relaxation (upper panel) of graphene sheet having monovacancy is shown, respectively. The dark solid line (black) indicates the average DOS for carbon atoms nearest neighbor to the vacancy (n.n. C), while the light solid line (red) shows the DOS for the $\mathrm{C}$ atoms occupying the next nearest neighbor positions from the vacancy (n.n.n. C). The vertical line represents the Fermi level which is fixed to zero. 
TABLE II. Relaxation of graphene with monovacancy constrained to $x y$ plane and free relaxation give two distinct Jahn-Teller distorted structures, which is shown for different total magnetic moment values $\left(M\right.$ in $\left.\mu_{\mathrm{B}}\right)$. The relaxed structural parameters with respect to Figure 2(a) are tabulated. The largest bond length ( $d$ in $\AA$ ) of the $\mathrm{C}-\mathrm{C}$ bond around the monovacancy and other two bond lengths referenced to the largest bond length are shown. The energy $(\Delta E$ in $\mathrm{eV})$ is shown with respect to the lowest energy obtained for respective type of relaxation.

\begin{tabular}{llllllllllll}
\hline \hline & \multicolumn{3}{c}{ Relaxation along $x y$ plane } & & \multicolumn{4}{c}{ Complete relaxation } \\
\cline { 2 - 3 }$M$ & $\Delta E$ & $d_{\mathbf{B C}}$ & $d_{\mathbf{A B}} / d_{\mathbf{B C}}$ & $d_{\mathbf{A C}} / d_{\mathbf{B C}}$ & & $\Delta E$ & $d_{\mathbf{A B}}$ & $d_{\mathbf{A C}} / d_{\mathbf{A B}}$ & $d_{\mathbf{B C}} / d_{\mathbf{A B}}$ \\
\hline 0.00 & 0.095 & 2.653 & 0.924 & 0.924 & & 0.116 & 2.612 & 0.990 & 0.800 \\
1.00 & 0.039 & 2.639 & 0.924 & 0.923 & & 0.030 & 2.599 & 0.990 & 0.786 \\
1.56 & 0.0 & 2.641 & 0.923 & 0.923 & & 0.0 & 2.600 & 0.990 & 0.791 \\
2.00 & 0.030 & 2.649 & 0.925 & 0.925 & & 0.050 & 2.611 & 0.990 & 0.896 \\
3.00 & 0.627 & 2.621 & 0.945 & 0.945 & & 0.732 & 2.610 & 0.989 & 0.803 \\
4.00 & 1.399 & 2.563 & 0.982 & 0.982 & & 1.685 & 2.498 & 0.990 & 0.800 \\
\hline \hline
\end{tabular}

relaxation with the atomic movement restricted to the $x y$ plane ( $x y$ relax) was done which gave a distorted triangle where the $\mathrm{C}-\mathrm{C}$ distance $d_{\mathbf{B C}}$ is larger than $d_{\mathbf{A B}}$ and $d_{\mathbf{A C}}$ with $d_{\mathbf{A B}}$ $=d_{\mathrm{AC}}$. However, we could find another JT distorted structure for defect graphene via relaxation with complete freedom to the atomic movements (complete relaxation). In the latter case $d_{\mathbf{B C}}$ was the shortest bond length. The energetics and bond lengths for both relaxation patterns are listed in Table II. The complete relaxed structure is about $3 \mathrm{meV} / \mathrm{atom}$ lower in total energy than the $x y$ relaxed structure. The complete relaxed structure is in agreement with other DFT studies ${ }^{31,54-56}$ and the structure of defect graphene has been observed in experiments under the high resolution transmission electron microscope; see Fig. 3(f) of Ref. 57. The electronic DOS of defective graphene for the two different JT distortions together with the unrelaxed case is shown in Fig. 2(b). The spin polarized DOS for unrelaxed defect graphene shows the states passing through the Fermi energy in both spin channels. After relaxation, the degeneracy is lifted due to the change in crystal field splittings which is a result of deviation from local symmetry due to reconstruction. The crystal field splitting appears more strongly on the nearest neighbor carbon atoms surrounding the vacancy (atoms marked A, B, and C in Fig. 2(a)) for both the relaxed geometry. The splitting is larger in case of the freely relaxed defective graphene.

Figure 2(b) also shows the exchange splitting between the majority and minority DOS due to the appearance of the magnetic moment for the complete relaxed defective graphene. The magnetic moment for the defective graphene sheet is found to be $1.60 \mu_{\mathrm{B}}$ /supercell, which is consistent with previous studies ${ }^{58}$ (the unrelaxed structure shows a magnetic moment of $1.18 \mu_{\mathrm{B}}$ /supercell). The magnetic moment is spread nonuniformly on the atomic sites, with the carbon atoms close to the vacancy site having the largest magnetic moment with $0.40 \mu_{\mathrm{B}}$, while the magnetic moment on $\mathrm{C}$ atoms decreases rapidly as the distance from the vacancy site is increased with the carbon atoms reasonably far away from defect site having no magnetic moment. The appearance of magnetic moment in defective graphene is important, especially for studies of TM
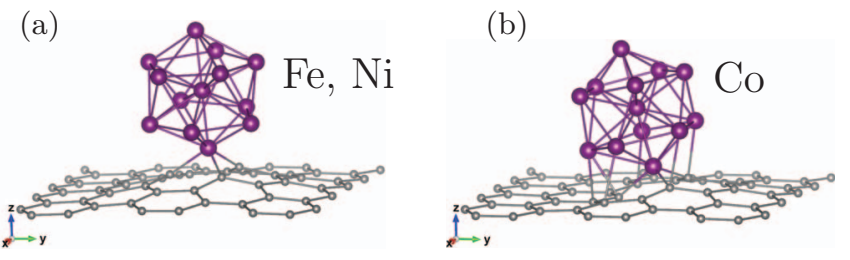

FIG. 3. Optimized structures of (a) $\mathrm{Fe}_{13}$ and $\mathrm{Ni}_{13}$, and (b) $\mathrm{Co}_{13}$ clusters adsorbed on graphene sheet with a monovacancy defect. The vertex atom of the icosahedral TM cluster is bonded to the $\mathrm{C}$ atoms surrounding the monovacancy. The dark (purple) balls and the light (grey) balls represent the TM and $\mathrm{C}$ atoms, respectively.

cluster adsorbed on defective graphene as the magnetic moment of defect graphene will interact and influence the magnetic moment of the TM clusters.

The $\mathrm{TM}_{13}$ clusters were deposited on to the graphene sheet with monovacancy defect and allowed to relax completely. Figure 3 shows the optimized structures of $\mathrm{TM}_{13}$ clusters adsorbed on graphene with monovacancy defect. While $\mathrm{Fe}_{13}$ and $\mathrm{Ni}_{13}$ are found to be adsorbed in an orientation where one of the vertex atoms of the icosahedral cluster is bonded with the $\mathrm{C}$ atoms surrounding the monovacancy site of defective graphene, $\mathrm{Co}_{13}$, on the other hand, relaxes to a tilted orientation owing to its tendency to maximize $\mathrm{Co}-\mathrm{C}$ bonds. The minimum and maximum bond lengths from the center to the surface atom in $\mathrm{Ni}_{13}$ cluster are $2.24 \AA$ and $2.51 \AA$, respectively, giving a variation $\left(\Delta d_{\text {Center-shell }}\right)$ of $0.27 \AA$. While, for $\mathrm{Fe}_{13}$ and $\mathrm{Co}_{13}$ the $\Delta d_{\text {Center-shell }}$ are $0.26 \AA$ and $0.22 \AA$, respectively. This tendency is opposite to that observed for $\mathrm{TM}_{13}$ adsorbed on pristine graphene where $\mathrm{Co}_{13}$ shows the maximum distortion (previously mentioned). The average centershell distances for all cases considered are listed in Table I. In addition, the $\mathrm{C}$ atoms in the substrate which are close to the clusters show local distortions and are pulled towards the cluster which is most notable for the $\mathrm{Co}_{13}$ adsorbed on defective graphene sheet.

The values for the $E_{\mathrm{Ads}}$ and magnetic moments for these clusters are shown in Table I. We observe significant enhancement in the $E_{\text {Ads }}$ for $\mathrm{TM}_{13}$ clusters on defective graphene as compared to that of the clusters adsorbed on pristine graphene because the dangling bonds of the three carbon atoms in the presence of a carbon vacancy are bonded to the $\mathrm{TM}_{13}$ cluster, thereby resulting in the stability and lowering the total energy. The $E_{\mathrm{Ads}}$ for $\mathrm{Co}_{13}$ is found to be more as compared to $\mathrm{Fe}_{13}$ and $\mathrm{Ni}_{13}$ adsorbed on defective graphene sheet as observed for $\mathrm{TM}_{13}$ adsorbed in pristine graphene sheet. This implies that $\mathrm{Co}_{13}$ is strongly bound to the defect graphene sheet. Our calculated value of $E_{\mathrm{Ads}}$ for $\mathrm{Fe}_{13}$ on defective graphene is consistent with previously reported value. ${ }^{31}$ We also observe a large charge transfer from cluster to graphene for the case of defective sheet which is $0.12 e\left(\mathrm{Fe}_{13}\right), 0.11 e\left(\mathrm{Co}_{13}\right)$, and $0.09 e\left(\mathrm{Ni}_{13}\right)$ as compared to that of the clusters adsorbed on pristine sheet which is $0.08 e, 0.09 e$, and $0.05 e$ for $\mathrm{Fe}_{13}, \mathrm{Co}_{13}$, and $\mathrm{Ni}_{13}$, respectively. The amounts of charge on both cluster and graphene are listed in Table I using the Bader charge analysis. ${ }^{59,60}$ The average Bader charges on clusters $\left\langle q_{\mathrm{TM}}^{\mathrm{B}}\right\rangle$ are calculated on the TM atoms close to the graphene substrate forming the nearest neighbor TM-C bonds, similarly, 
(a)

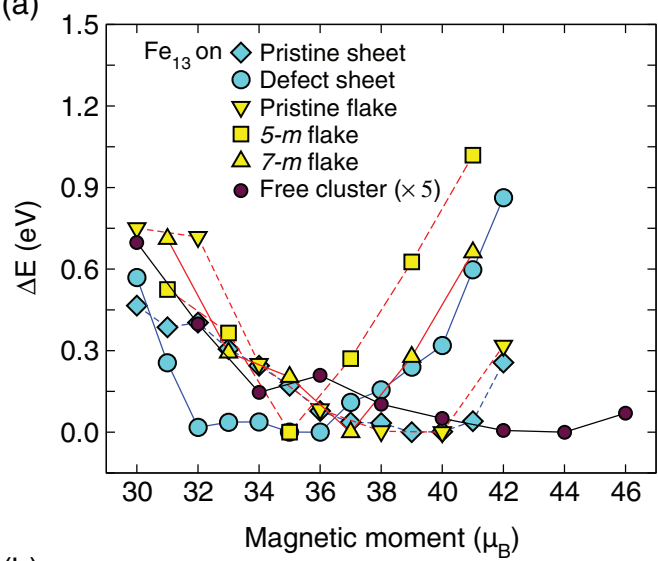

(b)

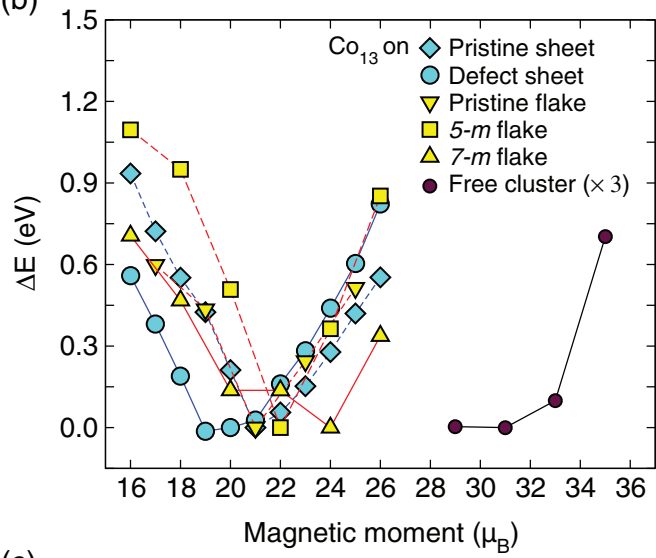

(c)

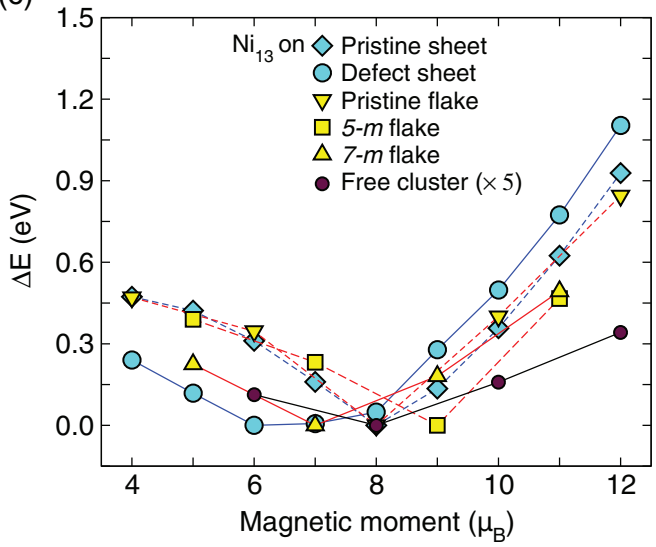

FIG. 4. Variation of the total energy with respect to minimum energy obtained for the adsorbed $\mathrm{Fe}_{13}(\mathrm{a}), \mathrm{Co}_{13}$ (b), and $\mathrm{Ni}_{13}$ (c) clusters as a function of fixed spin moment. The symbols diamond, circle, down triangle, square, and up triangle are denoted for pristine graphene sheet, graphene sheet with monovacancy, pristine graphene flake, 5- $m$ graphene flake, and 7- $m$ graphene flake, respectively. The light (yellow) and grey (cyan) fillings of the symbols represent cases for graphene sheet and graphene flakes, respectively. The black line for each case indicates the total energy differences for the free clusters which are multiplied by a factor of five, three, five, respectively, for $\mathrm{Fe}$, $\mathrm{Co}$, and $\mathrm{Ni}$, in order to match the $\mathrm{y}$-axis range.

the average charge on C-atoms $\left\langle q_{\mathrm{C}}^{\mathrm{B}}\right\rangle$ are calculated for those $\mathrm{C}$-atoms bonded to the TM atoms.

Figure 4 shows the energy difference $(\Delta E)$ obtained with respect to the minimum energy versus the spin moment for various cases of $\mathrm{TM}_{13}$ adsorbed on graphene using the fixed spin moment method. The diamond and circle compare the $\mathrm{TM}_{13}$ adsorption on pristine and defective graphene sheet, respectively. The magnetic moments of the adsorbed $\mathrm{TM}_{13}$ clusters on pristine and defective graphene are found to be reduced with respect to their corresponding free clusters for $\mathrm{Fe}$ and Co. For example, the total magnetic moment for adsorbed $\mathrm{Fe}_{13}$ on pristine graphene is found to be $39 \mu_{\mathrm{B}}$, which is $5 \mu_{\mathrm{B}}$ lower than the magnetic moment of free $\mathrm{Fe}_{13}$ cluster with value $44 \mu_{\mathrm{B}} /$ cluster. A larger reduction of magnetic moment is found for $\mathrm{Co}_{13}$ (where the total magnetic moment is reduced by $10 \mu_{\mathrm{B}}$, i.e., from $31 \mu_{\mathrm{B}}$ to $21 \mu_{\mathrm{B}}$ ), while for $\mathrm{Ni}_{13}$ the magnetic moment remains unchanged. The reduction of magnetic moment on $\mathrm{TM}_{13}$ adsorbed on defective graphene is relatively more than the corresponding pristine case, i.e., the total magnetic moment is reduced by $8 \mu_{\mathrm{B}}, 12 \mu_{\mathrm{B}}$, and $2 \mu_{\mathrm{B}}$ for $\mathrm{Fe}, \mathrm{Co}$, and Ni clusters, respectively. This is due to the fact that the dangling carbon atoms in the defect graphene are strongly hybridized with the $d$-states of the cluster atoms, thereby reducing the magnetic moment. The magnetic moment of the vertex atom for $\mathrm{Fe}_{13}$ and $\mathrm{Ni}_{13}$ adsorbed on defective sheet has lower magnitude. They are estimated to be 0.45 $\mu_{\mathrm{B}}$ and $0.05 \mu_{\mathrm{B}}$ in comparison to $2.75 \mu_{\mathrm{B}}$ and $0.53 \mu_{\mathrm{B}}$ for the average magnetic moment of rest twelve atoms for $\mathrm{Fe}_{13}$ and $\mathrm{Ni}_{13}$, respectively. In fact, the magnetic moments of $\mathrm{Ni}$ atoms vary from $0.44 \mu_{\mathrm{B}}$ to $0.80 \mu_{\mathrm{B}}$ (except the vertex atom facing graphene surface) depending on how far the atoms lie from the graphene surface. In case of $\mathrm{Co}_{13}$ cluster deposited on defective graphene, we find that two atoms which are close to the graphene surface have significantly low magnetic moment with values $0.12 \mu_{\mathrm{B}}$ and $1.01 \mu_{\mathrm{B}}$ which are aligned antiparallel to each other. The average magnetic moment per Co atom for other atoms in the cluster amount to $1.66 \mu_{\mathrm{B}}$. We observe induced magnetic moment on the $\mathrm{C}$ atoms in the graphene sheet. The induced magnetic moment has a value of $0.23 \mu_{\mathrm{B}}$ for adsorbed $\mathrm{Fe}_{13}, 0.12 \mu_{\mathrm{B}}$ for adsorbed $\mathrm{Co}_{13}$, and $0.05 \mu_{\mathrm{B}}$ for adsorbed $\mathrm{Ni}_{13}$ on graphene. Similar effects have been previously observed for adatoms adsorbed on graphene. ${ }^{27}$ The value of the magnetic moments for $\mathrm{TM}_{13}$ adsorbed on pristine and defective graphene are tabulated in Table I.

A recent theoretical study by Gao et al. ${ }^{61}$ for Ni clusters (up to nine atoms) adsorbed on monovacancy defect graphene has shown that the ground state geometry of Ni cluster is a configuration where one of the $\mathrm{Ni}$ atoms lies at the other side of graphene sheet than the rest of the atoms in the cluster. In order to check whether 13-atom icosahedral clusters also prefer such type of distribution of atoms as a low-energy structure, we performed additional calculations where we deliberately moved the vertex atom of the cluster facing the graphene sheet with monovacancy to the other side of the surface. Thus one adatom and a twelve atom cluster lie on two sides of the graphene sheet facing each other through the monovacancy defect. After performing the structural optimization, the total energies are compared with the case of 13-atom icosahedral cluster adsorbed on one side of the defected graphene sheet which have been systematically used in the present study. We find that clusters on one side of the graphene sheet are energetically more favorable than the case where one atom of the cluster lies on the opposite side of the sheet. The energy difference amounts to $84.41 \mathrm{meV} / \mathrm{atom}, 33.36 \mathrm{meV} /$ atom, and $19.62 \mathrm{meV} /$ atom for $\mathrm{Fe}, \mathrm{Co}$, and $\mathrm{Ni}$, respectively. The relaxed structures of TM clusters with one of the TM atoms lying on 
the opposite side of graphene surface show that the adatom is bonded to the three $\mathrm{C}$ atoms of the substrate surrounding the monovacancy. The relaxation of rest twelve atoms is significant for $\mathrm{Ni}$ which undergoes a lateral displacement such that one of the vertex atoms is bonded to the three carbon atoms surrounding the monovacancy. This leads to the formation of one $\mathrm{Ni}-\mathrm{Ni}$ bond between the $\mathrm{Ni}$ atoms placed on the opposite side of the graphene sheet through the vacancy region. This is unlike the $\mathrm{Fe}$ and Co clusters, where the twelve atom clusters were observed to have closed geometry. While the total magnetic moment of $\mathrm{Co}_{13}$ cluster did not change, the total magnetic moment of $\mathrm{Fe}_{13}$ cluster is reduced by $4 \mu_{\mathrm{B}}$ to a value of $32 \mu_{\mathrm{B}}$, while the total magnetic moment of $\mathrm{Ni}_{13}$ cluster is increased by $2 \mu_{\mathrm{B}}$ to give $8 \mu_{\mathrm{B}}$ (compare the total magnetic moment for the cluster adsorbed on one side of defective graphene sheet from Table I).

\section{Clusters on 5- $m$ and 7- $m$ graphene flakes}

In addition to clusters supported on graphene sheet, we have studied the adsorption of $\mathrm{TM}_{13}$ clusters on graphene flakes. The graphene flakes consist of finite chunks of graphene and are passivated with $\mathrm{H}$ at the edge carbon atoms, which we name as pristine graphene flake. In order to study the role of defects in the graphene flakes on the adsorption of $\mathrm{TM}_{13}$ clusters, we consider the pentagonal and heptagonal structural defects, namely, 5- $m$ and 7-m graphene flakes. The hydrocarbon clusters with $\mathrm{C}_{54} \mathrm{H}_{18}, \mathrm{C}_{45} \mathrm{H}_{15}$, and $\mathrm{C}_{63} \mathrm{H}_{21}$ are considered in our studies for the pristine, $5-m$ and $7-m$ graphene flakes, respectively.

For clusters on pristine graphene flake, the optimized structures of $\mathrm{Fe}_{13}$ and $\mathrm{Ni}_{13}$ are shown in Fig. 5(a) (owing to the similarity in geometry, they are shown in one figure) and that of the $\mathrm{Co}_{13}$ in Fig. 5(b). The structural configurations are similar to that of the respective $\mathrm{TM}_{13}$ clusters adsorbed on pristine graphene sheet (refer Fig. 1). Similar to that of the pristine graphene sheet, $\mathrm{Co}_{13}$ is adsorbed on pristine graphene flake with an orientation where one of the Co atoms is bonded to six $\mathrm{C}$ atoms of a hexagonal ring of the graphene flake. As a result, the magnetic moment of the Co atom is reduced to a value $0.63 \mu_{\mathrm{B}}$ as compared to the average magnetic moment of other twelve Co atoms as $1.69 \mu_{\mathrm{B}}$ /atom. Such drastic reduction of atomic moment of one of the atoms is not observed for $\mathrm{Fe}_{13}$ adsorbed on graphene flake, while for $\mathrm{Ni}_{13}$, the atomic magnetic moment varies from $0.35 \mu_{\mathrm{B}}$ to $0.83 \mu_{\mathrm{B}}-$ the farther the $\mathrm{Ni}$ atom from the surface of graphene flake, the larger is the magnetic moment. These tendencies are similar to $\mathrm{TM}_{13}$ adsorbed on pristine graphene sheet. The $E_{\mathrm{Ads}}$ and magnetic moments of these clusters on both 5- $m$ and 7-m graphene flakes are listed in Table I. It shows that the $E_{\mathrm{Ads}}$ for $\mathrm{TM}_{13}$ clusters is enhanced for the defect graphene flakes compared to that of the pristine graphene flake. The optimized structures for clusters on graphene flakes (pristine and defective) are shown in Fig. 5. $\mathrm{TM}_{13}$ clusters on 5-m graphene flake have a large binding strength compared to those of on 7-m graphene flake for $\mathrm{Fe}$ and $\mathrm{Co}$. Similar trends have been observed from DFT for $\mathrm{Pt}_{13}$ and $\mathrm{Au}_{13}$ clusters adsorbed on 5-m and 7- $m$ graphene flakes. ${ }^{62}$ However, $\mathrm{Ni}_{13}$ shows an opposite behavior where the $E_{\mathrm{Ads}}$ of $\mathrm{Ni}_{13}$ on 7- $m$ graphene flake is en-
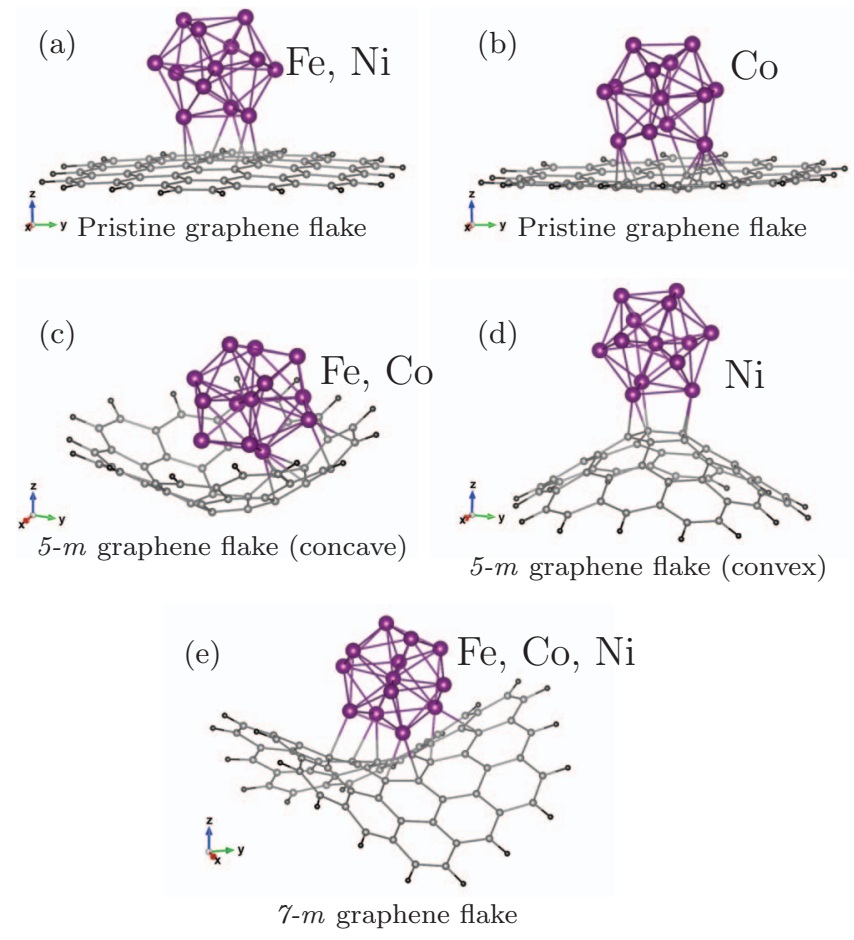

FIG. 5. The optimized structures of (a) $\mathrm{Fe}_{13}, \mathrm{Ni}_{13}$ and (b) $\mathrm{Co}_{13}$ on pristine graphene flake. The low energy structures of $\mathrm{Fe}_{13}$ and $\mathrm{Co}_{13}$ adsorbed on 5-m graphene flake are shown in (c), while the low energy structures of $\mathrm{Ni}_{13}$ adsorbed on 5- $m$ graphene flake are shown in (d). It must be noted that while $\mathrm{Fe}_{13}$ and $\mathrm{Co}_{13}$ favor a concave structure as in (c), $\mathrm{Ni}_{13}$ favors convex structure when adsorbed on 5- $m$ graphene flake. The optimized structures of $\mathrm{Fe}_{13}$, $\mathrm{Co}_{13}$, and $\mathrm{Ni}_{13}$ on 7-m graphene flake are shown in (e). Owing to the similarity in optimized structures, only one figure is used for representation. The larger dark shaded balls (purple), the light balls (grey), and smaller dark balls (black) represent the TM, C, and the passivating hydrogen atoms, respectively.

hanced relative to the 5- $m$ graphene flake. This is attributed to the different structural changes and the bending behavior of the 5- $\mathrm{m}$ graphene flakes. The adsorption of $\mathrm{TM}_{13}$ clusters on 5- $m$ graphene flake results in two different bending trends, namely, the concave geometry (where the 5- $m$ graphene flake bends towards the cluster allowing for maximum coordination of the carbon atoms) and the convex geometry (where the $5-\mathrm{m}$ graphene flake bends away from the cluster). The energetics and magnetic moments for the concave and convex geometries of $\mathrm{TM}_{13}$ clusters are listed in Table III. For $\mathrm{Fe}_{13}$ and $\mathrm{Co}_{13}$, it is found that the concave geometry is energetically more favorable, whereas for $\mathrm{Ni}_{13}$ the convex geometry is more favorable. Moreover, a reduction in the total magnetic moment is observed for $\mathrm{Fe}_{13}$ and $\mathrm{Co}_{13}$ clusters on 5-m graphene flake, whereas for $\mathrm{Ni}_{13}$ the moment remains unchanged with respect to the free $\mathrm{Ni}_{13}$ cluster. Among the adsorbed clusters on graphene flakes, $\mathrm{Fe}_{13}$ and $\mathrm{Co}_{13}$ are strongly bound to the 5- $m$ graphene flake with the $E_{\mathrm{Ads}}$ values of $2.81 \mathrm{eV}$ and 4.31 $\mathrm{eV}$, respectively, while $\mathrm{Ni}_{13}$ is strongly bound to $7-m$ graphene flake with binding energy of $3.73 \mathrm{eV}$. The optimized geometry of $\mathrm{TM}_{13}$ on 7- $m$ graphene flake is similar, which is shown in Fig. 5(e). Due to the topology of the 7- $m$ graphene flake, there is a possibility for the higher coordination of $\mathrm{C}$ atoms around the TM atoms. We find that the average nearest neighbor bond length of $\mathrm{Fe}-\mathrm{C}, \mathrm{Co}-\mathrm{C}$, and $\mathrm{Ni}-\mathrm{C}$ are $2.18 \AA, 2.10 \AA$, and $2.05 \AA$, respectively. 
TABLE III. The total energy differences $\left(\Delta E=E_{\text {Concave }}-E_{\text {Convex }}\right)$ in $\mathrm{eV}$ for the relaxed low energy structures and their corresponding magnetic moments $\left(\mu_{\mathrm{B}}\right)$ for $\mathrm{TM}_{13}$ clusters on concave and convex structures of 5-m graphene flake as represented in Figs. 5(c) and 5(d), respectively. $\left\langle d_{\text {Ring }}\right\rangle$ is the average $\mathrm{C}-\mathrm{C}$ bondlength (defined as the average over six $\mathrm{C}$ atoms in the ring) in the 5- $m$ graphene flake.

\begin{tabular}{lrrrrrr}
\hline \hline & & \multicolumn{2}{c}{ Concave } & & \multicolumn{2}{c}{ Convex } \\
\cline { 3 - 4 } \cline { 6 - 7 } Cluster & \multicolumn{1}{c}{$\Delta E$} & $\left\langle d_{\text {Ring }}\right\rangle$ & $\mathrm{M}$ & & $\left\langle d_{\text {Ring }}\right\rangle$ & $\mathrm{M}$ \\
\hline $\mathrm{Fe}_{13}$ & -0.435 & 1.429 & 35.0 & & 1.455 & 39.0 \\
$\mathrm{Co}_{13}$ & -1.159 & 1.425 & 22.0 & & 1.461 & 24.0 \\
$\mathrm{Ni}_{13}$ & 0.105 & 1.425 & 9.0 & & 1.455 & 9.0 \\
\hline \hline
\end{tabular}

The plots in Fig. 4 show that the magnetic moment of $\mathrm{TM}_{13}$ clusters on defect sheet can be sensitive to the surrounding environment and the external magnetic fields as the energy differences for slight deviation of magnetic moment from the low energy magnetic states are rather low. Fe allows for the change in magnetic moment of about $4 \mu_{\mathrm{B}}\left(32 \mu_{\mathrm{B}}\right.$ /cluster to $36 \mu_{\mathrm{B}}$ /cluster, roughly), similarly, Co allows for $2 \mu_{\mathrm{B}}$ $\left(19 \mu_{\mathrm{B}} /\right.$ cluster to $21 \mu_{\mathrm{B}}$ /cluster, roughly) and Ni allows $2 \mu_{\mathrm{B}}$ (6 $\mu_{\mathrm{B}} /$ cluster and $8 \mu_{\mathrm{B}} /$ cluster, roughly). On the other hand, the magnetic moment changes for TM clusters deposited on pristine and defective graphene flakes are associated with large changes in total energies. This suggests that the TM cluster-graphene flake system can be treated as system with giant spins. Large magnetic moment and high magnetic anisotropy are requisite for technological applications. Keeping this in mind, we calculated the magneto-crystalline anisotropy by considering the contribution due to spin-orbit coupling. We have obtained magnetic anisotropy energy of $1.126 \mathrm{meV}$ for $\mathrm{Ni}_{13}$ cluster on convex 5-m graphene flake, which is several orders of magnitude higher than $\mathrm{Ni}$ bulk (2.7 $\mu \mathrm{eV} /$ atom). The orbital moments along the easy and hard axes are found to be $0.98 \mu_{\mathrm{B}}$ and $0.96 \mu_{\mathrm{B}}$, respectively. Bader charge $\left(q^{\mathrm{B}}\right)$ analysis of average atomic charges on the TM atom and on $\mathrm{C}$ atom shows that there is a transfer of electronic charges from the TM atoms of cluster to the $\mathrm{C}$ atoms of graphene. The results are tabulated in Table I. It is observed that the amounts of charge transfer are slightly increased for $\mathrm{Fe}$ and Co clusters adsorbed on 5- $m$ and 7- $m$ graphene flakes as compared to $\mathrm{Fe}$ and Co clusters adsorbed on pristine graphene flake. An opposite tendency is observed in case of Ni cluster adsorbed on 5- $m$ and 7- $m$ graphene flakes as compared to $\mathrm{Ni}$ cluster adsorbed to pristine graphene flake where the charge transfer decreases. For all the cases of TM adsorbed on various types of graphene substrates, the $E_{\mathrm{Ads}}$ is found to be proportional to the charge transfer from the TM atoms of the cluster to the carbon atoms of graphene. As observed from Table I, we note that although the $E_{\mathrm{Ads}}$ varies in the order of $E_{\mathrm{Ads}}$ (pristine flake) $<E_{\mathrm{Ads}}(7-m$ flake $)<E_{\mathrm{Ads}}$ (5- $m$ flake), for $\mathrm{Fe}$ and Co clusters, the magnetic moments do not follow similar trend. This is because of the structural orientation of $\mathrm{Co}_{13}$, where the vertex Co atom has very low magnetic moment due to the bonding with six $\mathrm{C}$ atoms form a hexagonal ring of pristine graphene flake. Similar structural orientation is also found for $\mathrm{Co}_{13}$ adsorbed on pristine graphene sheet, which is already discussed.

In order to analyze the variations in the magnetic moment for $\mathrm{Fe}_{13}, \mathrm{Co}_{13}$, and $\mathrm{Ni}_{13}$ clusters deposited on the 5-m graphene flake between the two curvatures and the preference of the convex shape for $\mathrm{Ni}_{13}$, we have plotted the site and the orbital projected DOS for the lowest energy structures for $\mathrm{Fe}_{13}$ and $\mathrm{Co}_{13}$ (cluster on concave 5- $m$ graphene flake) in Fig. 6. For $\mathrm{Ni}_{13}$, the corresponding DOS for the concave and convex configurations are plotted in Fig. 7. We first consider the concave geometries for all the clusters. Note that the mixing between the $\mathrm{C} p$-states and the TM $d$-states changes with the elemental species of the cluster. The mixing is strongest for $\mathrm{Co}_{13}$ and weakest in case of $\mathrm{Ni}_{13}$. In fact, the width of the $d$-states is the lowest for the case of $\mathrm{Ni}_{13}$. A change from
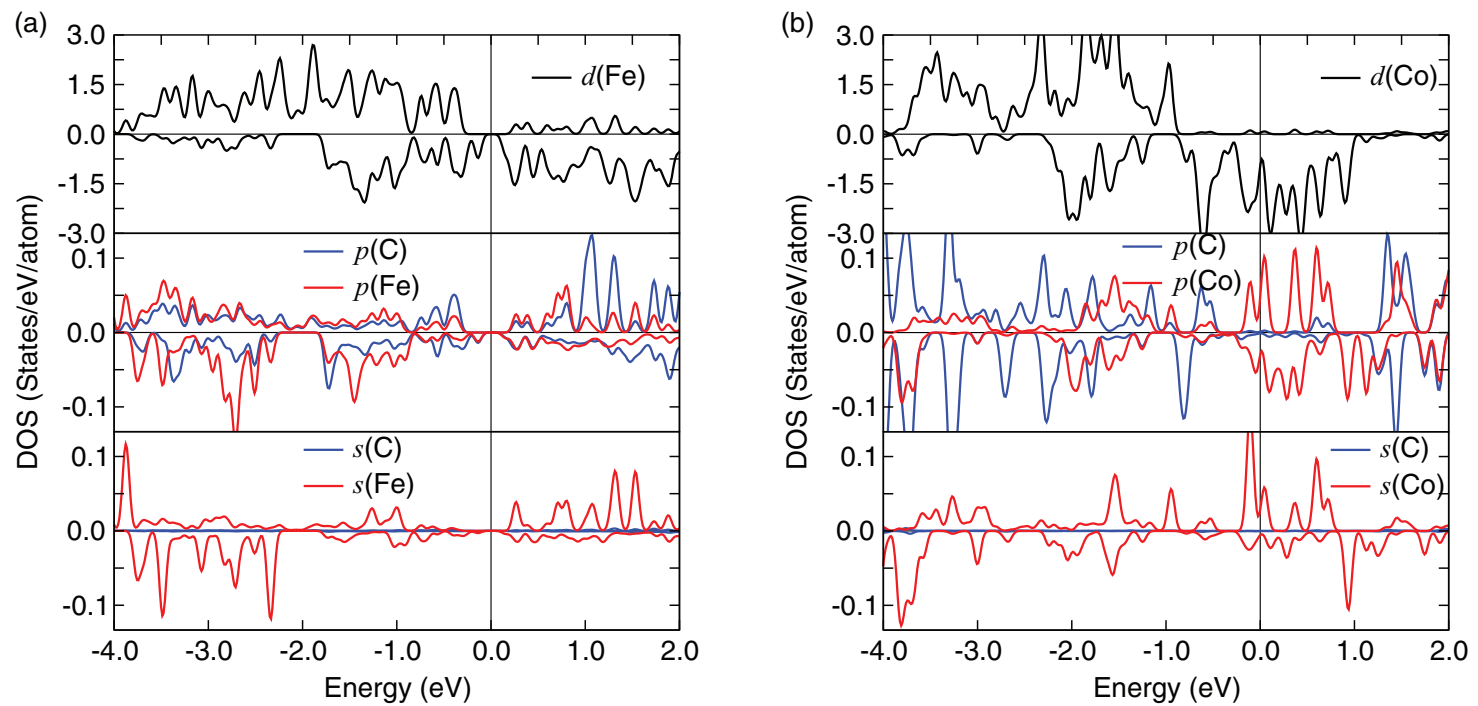

FIG. 6. Site and orbital projected average DOS of elemental species for $\mathrm{Fe}_{13}$ (a) and $\mathrm{Co}_{13}$ (b) on concave 5 - $m$ graphene flake (as shown in Fig. 5 (c)), respectively. The average $d$-orbital contribution of the TM atoms is shown as black solid line in the upper panels, the dark (blue) and light (red) solid lines show the average $p$-orbital contribution from $\mathrm{C}$ and TM atoms in the middle panel, and the dark (blue color) and light (red color) solid lines show the average $s$-orbital contribution from C and TM atoms in the lower panel, respectively. The vertical lines represent the Fermi level which is set to zero. 

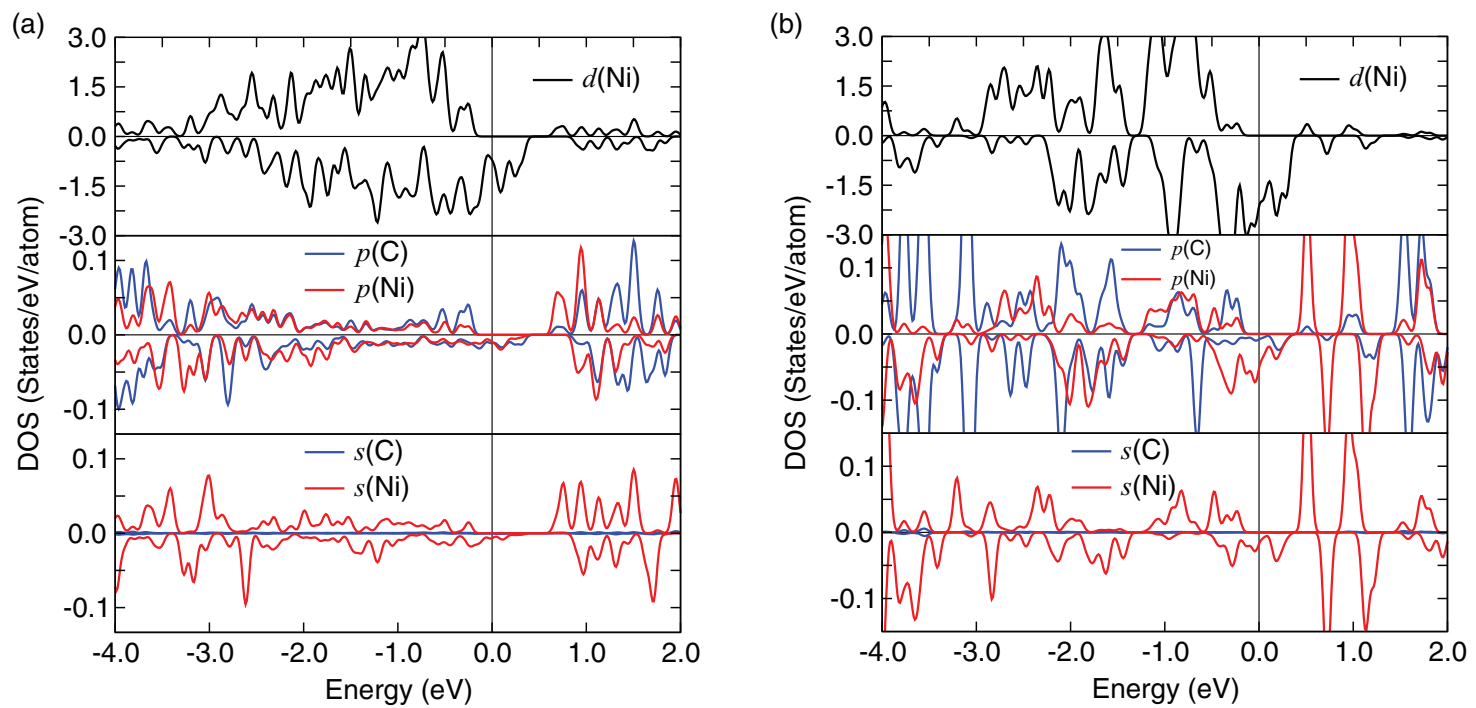

FIG. 7. Site and orbital projected average DOS of elemental species for $\mathrm{Ni}_{13}$ on concave (a) and convex (b) 5- $m$ graphene flake (as shown in Fig. 5 (d)), respectively. The average $d$-orbital contribution of the TM atoms is shown as black solid line in the upper panels, the dark (blue) and light (red) solid lines show the average $p$-orbital contribution from C and TM atoms in the middle panel, and the dark (blue) and light (red) solid lines show the average $s$-orbital contribution from $\mathrm{C}$ and TM atoms in the lower panel, respectively. The vertical lines represent the Fermi level which is set to zero.

convex to concave bending is expected to alter the mixing between the carbon $p$-states and the TM $d$-states (carbon $s$-states are deeper in energy). In particular, any change in the mixing with the $d$-states is expected to change the magnetic moment. In view of the large hybridization, the change is expected to be maximum for the case of $\mathrm{Co}_{13}$ and lowest for the case of $\mathrm{Ni}_{13}$. Our calculated values indeed confirm this as $\mathrm{Co}_{13}$ undergoes a change in moment by $4 \mu_{\mathrm{B}}$ as opposed to $2 \mu_{\mathrm{B}}$ for the case of $\mathrm{Fe}_{13}$ and no change in the case of $\mathrm{Ni}_{13}$. Figure 7 also shows that the convex shape allows a slightly better mixing between the $\mathrm{C} p$-states and $\mathrm{Ni} d$-states than the concave shape. The better mixing is consistent with the change in curvature to convex curvature for the case of $\mathrm{Ni}_{13}$.

\section{SUMMARY}

To summarize, we have examined the suitability of pristine graphene, defected graphene, pristine graphene flakes, and defected graphene flakes as support for transition metal clusters. Our investigations have been motivated by the fact that free clusters, while interesting, need to be supported for practical applications. The present investigations bring out three important features. First, the clusters are more strongly bound to the defected sheets and flakes indicating that the defected systems are needed for stabilizing the clusters. Second, the binding to the defected graphene (both sheets and flakes) does not reduce the cluster magnetic moment significantly compared to that of those on pristine sheet. For $\mathrm{Fe}_{13}$, the magnetic moment of the cluster deposited on 5- $m$ graphene flake is $35 \mu_{\mathrm{B}}$ only slightly reduced from the cluster deposited on pristine sheet, i.e., $39 \mu_{\mathrm{B}}$. On the other hand, $\mathrm{Co}_{13}$ and $\mathrm{Ni}_{13}$ undergo an enhancement of the magnetic moment as compared to that of the pristine sheet of values of $21 \mu_{\mathrm{B}} /$ atom and $8 \mu_{\mathrm{B}} /$ atom to $22 \mu_{\mathrm{B}} /$ atom and $9 \mu_{\mathrm{B}} /$ atom, respectively, even though the deposition entails binding with carbon sites. Finally, and surprisingly, the cluster deposition in the case of
$\mathrm{Ni}_{13}$ on 5- $m$ graphene flake leads to a significant enhancement of the magnetic anisotropy by a factor of $\sim 420$ times compared to the bulk value of $2.7 \mu \mathrm{eV} / \mathrm{atom}$. This is particularly surprising since the conventional approach to enhance anisotropy is to deposit clusters on substrates such as $\mathrm{Pt}$ marked by high spin-orbit coupling. Here, the enhancement is mediated by carbon that is non magnetic with no significant spin-orbit coupling. One of the quantities of interest is the robustness of the magnetic moments. Figure 4 shows that the magnetic states on the 5- $m$ graphene flakes are fairly robust as any change in magnetic moment involves a larger change in energy of the system. We hope that the present work will stimulate the experimental studies for clusters on graphene sheets and flakes to investigate the changes in magnetic moments and magnetic anisotropies.

\section{ACKNOWLEDGMENTS}

Computational resources available from the Centre for Computational Sciences and Simulation (CCSS), University of Duisburg-Essen, is duly acknowledged. S.S. and S.N.K. gratefully acknowledge the support from U.S. Department of Energy (DOE) through Grant No. DE-FG02-11ER16213.

${ }^{1}$ A. Stiles, Catalyst Supports and Supported Catalysts: Theoretical and Applied Concepts (Butterworths, 1987).

${ }^{2}$ A. V. Melechko, V. I. Merkulov, T. E. McKnight, M. A. Guillorn, K. L. Klein, D. H. Lowndes, and M. L. Simpson, J. Appl. Phys. 97, 041301 (2005).

${ }^{3}$ E. Auer, A. Freund, J. Pietsch, and T. Tacke, Appl. Catal. A 173, 259 (1998).

${ }^{4}$ P. R. Wallace, Phys. Rev. 71, 622 (1947).

${ }^{5}$ A. H. Castro Neto, F. Guinea, N. M. R. Peres, K. S. Novoselov, and A. K. Geim, Rev. Mod. Phys. 81, 109 (2009).

${ }^{6}$ A. K. Geim and K. S. Novoselov, Nat. Mater. 6, 183 (2007).

${ }^{7}$ T. J. Echtermeyer, M. C. Lemme, J. Bolten, M. Baus, M. Ramsteiner, and H. Kurz, Eur. Phys. J. Special Topics 148, 19 (2007).

${ }^{8}$ K. J. Tielrooij, J. C. W. Song, S. A. Jensen, A. Centeno, A. Pesquera, A. Zurutuza Elorza, M. Bonn, L. S. Levitov, and F. H. L. Koppens, Nat. Phys. 9, 248 (2013). 
${ }^{9}$ C. J. Docherty, C.-T. Lin, H. J. Joyce, R. J. Nicholas, L. M. Herz, L.-J. Li, and M. B. Johnston, Nat. Commun. 3, 1228 (2012).

${ }^{10}$ E. Yoo, T. Okata, T. Akita, M. Kohyama, J. Nakamura, and I. Honma, Nano Lett. 9, 2255 (2009).

${ }^{11}$ M. Zhou, A. Zhang, Z. Dai, C. Zhang, and Y. P. Feng, J. Chem. Phys. 132, 194704 (2010).

${ }^{12}$ K. Yamamoto, T. Imaoka, W.-J. Chun, O. Enoki, H. Katoh, M. Takenaga, and A. Sonoi, Nat. Chem. 1, 397 (2009).

${ }^{13}$ Y. Li, Z. Zhou, G. Yu, W. Chen, and Z. Chen, J. Phys. Chem. C 114, 6250 (2010).

${ }^{14}$ E. H. Song, Z. Wen, and Q. Jiang, J. Phys. Chem. C 115, 3678 (2011).

${ }^{15}$ E. Ochoa-Fernández, D. Chen, Z. Yu, B. Tøtdal, M. Rønning, and A. Holmen, Surf. Sci. 554, L107 (2004).

${ }^{16}$ Z.-J. Liu, Z. Xu, Z.-Y. Yuan, D. Lu, W. Chen, and W. Zhou, Catal. Lett. 72, 203 (2001).

${ }^{17}$ H. Kim, W. Lee, and D. Yoo, Electrochim. Acta 52, 2620 (2007).

${ }^{18}$ C. A. Bessel, K. Laubernds, N. M. Rodriguez, and R. T. K. Baker, J. Phys. Chem. B 105, 1115 (2001).

${ }^{19}$ V. Selvaraj and M. Alagar, Electrochem. Commun. 9, 1145 (2007).

${ }^{20}$ Z. Liu, X. Y. Ling, B. Guo, L. Hong, and J. Y. Lee, J. Power Sources 167, 272 (2007).

${ }^{21}$ S. Kim and S.-J. Park, J. Solid State Electrochem. 11, 821 (2007).

${ }^{22}$ J.-S. Zheng, X.-S. Zhang, P. Li, J. Zhu, X.-G. Zhou, and W.-K. Yuan, Electrochem. Commun. 9, 895 (2007).

${ }^{23}$ R. Giordano, P. Serp, P. Kalck, Y. Kihn, J. Schreiber, C. Marhic, and J.-L. Duvail, Eur. J. Inorg. Chem. 2003, 610 (2003).

${ }^{24}$ R. Xiao, D. Fritsch, M. D. Kuz'min, K. Koepernik, H. Eschrig, M. Richter, K. Vietze, and G. Seifert, Phys. Rev. Lett. 103, 187201 (2009).

${ }^{25}$ S. Sahoo, A. Hucht, M. E. Gruner, G. Rollmann, P. Entel, A. Postnikov, J. Ferrer, L. Fernández-Seivane, M. Richter, D. Fritsch, and S. Sil, Phys. Rev. B 82, 054418 (2010).

${ }^{26}$ T. O. Wehling, A. V. Balatsky, M. I. Katsnelson, A. I. Lichtenstein, and A. Rosch, Phys. Rev. B 81, 115427 (2010).

${ }^{27}$ H. Johll, H. C. Kang, and E. S. Tok, Phys. Rev. B 79, 245416 (2009).

${ }^{28}$ C. Cao, M. Wu, J. Jiang, and H.-P. Cheng, Phys. Rev. B 81, 205424 (2010).

${ }^{29}$ K. T. Chan, J. B. Neaton, and M. L. Cohen, Phys. Rev. B 77, 235430 (2008).

${ }^{30}$ H. Johll, J. Wu, S. W. Ong, H. C. Kang, and E. S. Tok, Phys. Rev. B 83, 205408 (2011)

${ }^{31}$ D.-H. Lim, A. S. Negreira, and J. Wilcox, J. Phys. Chem. C 115, 8961 (2011).

${ }^{32}$ R. C. Longo, J. Carrete, J. Ferrer, and L. J. Gallego, Phys. Rev. B 81, 115418 (2010).

${ }^{33}$ J. Kotakoski, A. V. Krasheninnikov, U. Kaiser, and J. C. Meyer, Phys. Rev. Lett. 106, 105505 (2011).
${ }^{34}$ L. Li, S. Reich, and J. Robertson, Phys. Rev. B 72, 184109 (2005).

${ }^{35}$ J. M. Carlsson and M. Scheffler, Phys. Rev. Lett. 96, 046806 (2006).

${ }^{36}$ X. Y. Cui, R. K. Zheng, Z. W. Liu, L. Li, B. Delley, C. Stampfl, and S. P. Ringer, Phys. Rev. B 84, 125410 (2011).

${ }^{37}$ D. W. Boukhvalov and M. I. Katsnelson, Nano Lett. 8, 4373 (2008).

${ }^{38}$ H. Wang, J. T. Robinson, G. Diankov, and H. Dai, J. Am. Chem. Soc. 132, 3270 (2010).

${ }^{39}$ P. Kim, Nat. Mater. 9, 792 (2010).

${ }^{40}$ A. Stone and D. Wales, Chem. Phys. Lett. 128, 501 (1986).

${ }^{41}$ J. Kotakoski, J. C. Meyer, S. Kurasch, D. Santos-Cottin, U. Kaiser, and A. V. Krasheninnikov, Phys. Rev. B 83, 245420 (2011).

${ }^{42}$ G.-D. Lee, C. Z. Wang, E. Yoon, N.-M. Hwang, D.-Y. Kim, and K. M. Ho, Phys. Rev. Lett. 95, 205501 (2005).

${ }^{43}$ J. P. Perdew, K. Burke, and M. Ernzerhof, Phys. Rev. Lett. 77, 3865 (1996).

${ }^{44}$ G. Kresse and J. Furthmüller, Comput. Mater. Sci. 6, 15 (1996).

${ }^{45}$ G. Kresse and D. Joubert, Phys. Rev. B 59, 1758 (1999).

${ }^{46}$ P. E. Blöchl, Phys. Rev. B 50, 17953 (1994).

${ }^{47}$ O. Diéguez, M. M. G. Alemany, C. Rey, P. Ordejón, and L. J. Gallego, Phys. Rev. B 63, 205407 (2001).

${ }^{48}$ H. Duan and Q. Zheng, Phys. Lett. A 280, 333 (2001)

${ }^{49}$ G. Rollmann, M. E. Gruner, A. Hucht, R. Meyer, P. Entel, M. L. Tiago, and J. R. Chelikowsky, Phys. Rev. Lett. 99, 083402 (2007).

${ }^{50}$ M. E. Gruner and P. Entel, J. Phys.: Condens. Matter 21, 293201 (2009).

${ }^{51}$ G. Rollmann, P. Entel, and S. Sahoo, Comput. Mater. Sci. 35, 275 (2006).

${ }^{52}$ S. Sahoo, "Ab initio study of free and deposited transition metal clusters," Ph.D. thesis, University of Duisburg-Essen, 2011.

${ }^{53}$ H. A. Jahn and E. Teller, Proc. R. Soc. London A 161, 220 (1937).

${ }^{54}$ A. A. El-Barbary, R. H. Telling, C. P. Ewels, M. I. Heggie, and P. R. Briddon, Phys. Rev. B 68, 144107 (2003).

${ }^{55}$ Y. Ma, P. O. Lehtinen, A. S. Foster, and R. M. Nieminen, New J. Phys. 6, 68 (2004).

${ }^{56}$ B. R. K. Nanda, M. Sherafati, Z. S. Popovi, and S. Satpathy, New J. Phys. 14, 083004 (2012).

${ }^{57}$ J. C. Meyer, C. Kisielowski, R. Erni, M. D. Rossell, M. F. Crommie, and A. Zettl, Nano Lett. 8, 3582 (2008).

${ }^{58}$ V. V. Nelayev and A. I. Mironchik, Mater. Phys. Mech. 9, 26 (2010).

${ }^{59}$ R. F. W. Bader, Atoms in Molecules - A Quantum Theory (Oxford University Press, 1994).

${ }^{60}$ W. Tang, E. Sanville, and G. Henkelman, J. Phys.: Condens. Matter 21, 084204 (2009).

${ }^{61}$ W. Gao, J. E. Mueller, J. Anton, Q. Jiang, and T. Jacob, Angew. Chem. Int. Ed. 52, 14237 (2013).

${ }^{62}$ Y. Okamoto, Chem. Phys. Lett. 420, 382 (2006). 